\title{
An AC OPF-based Heuristic Algorithm for Optimal Transmission Switching
}

\author{
Florin Capitanescu* and Louis Wehenkel ${ }^{\dagger}$ \\ *Independent researcher \\ Email: fcapitanescu@yahoo.com \\ $\dagger$ Department of Electrical Engineering and Computer Science \\ University of Liège, B4000 Liège, Belgium \\ Email: l.wehenkel@ulg.ac.be
}

\begin{abstract}
This paper focuses on reducing generators dispatch cost by means of transmission line switching. The problem is formulated as a mixed-integer nonlinear program (MINLP) optimal power flow (OPF). A scalable heuristic algorithm is proposed to break-down the complexity of the problem due to the huge combinatorial space. The algorithm aims at providing the sequence of lines to be removed from service, one at the time, until no further decrease in the dispatch cost can be obtained. It identifies the line candidate for removal at each step by exploiting the (continuously relaxed values of) lines breaker statuses at the solution of a relaxed OPF problem. The algorithm thus relies on solving a sequence of OPF problems formulated as nonlinear programs (NLPs). The effectiveness of the approach is demonstrated on the IEEE118-bus system. Results show that the approach can provide good quality sub-optimal solutions with relatively small computational effort and by removing only few lines from service.
\end{abstract}

\section{INTRODUCTION}

Network switching (e.g., connection or disconnection of transmission lines, coupling or splitting of busbars, etc.) is a very low cost and sometimes very effective control means in power systems operation [1], [2], [3]. Network switching operations have been studied in the context of Optimal Power Flow (OPF) problems for various purposes (e.g., overload removal [4], [5], undervoltages/overvoltages alleviation [5], power loss reduction [6], [7], N-1 security improvement [8], generation dispatch cost reduction [3], [9], [10], [11], [12], [13], [14], etc.). The survey papers [1], [2], [3] provide extensive literature on this topic.

Optimal transmission switching (OTS) for generation dispatch cost reduction, which is the focus of the present paper, is a major research framework nowadays [3], [9], [10], [11], [12], [13], [14]. Because handling binary variables which model network switching within the non-convex and nonlinear AC model of a power system leads to a highly challenging large scale mixed integer nonlinear programming problem (MINLP), most previous works adopt the linear DC approximation [9], [10], [11], [12] and formulate the problem as a mixed-integer linear program (MILP). These works address mostly the challenges of solving MILPs with a large number of binary variables, while ignoring the intuitive fact that, the more lines are removed from service the less reliable the DC approximation is. Furthermore, as no AC OPF is considered for post-processing MILP results, these works do not guarantee that the topology obtained by the MILP approach is feasible (i.e. satisfies all network constraints, especially as the DC model neglects voltage variations and reactive power flows) nor that it indeed reduces the dispatch cost of the true nonlinear real-world problem.

To overcome these limitations, the OTS problem has been recently addressed while relying on adequate AC OPF models, which take into account voltage variations and reactive power aspects [13], [14]. Ref. [13] examines the impact on losses and voltage level obtained using the DC-based heuristic proposed in [11], and discusses the validity of the DC model. Ref. [14] assesses, using an exact AC OPF model, the quality of a DCbased OPF heuristic technique proposed in [11] for ranking the candidate lines to be removed from service.

The main contribution of the present paper is to propose a scalable AC OPF-based heuristic algorithm, aimed to breakdown the complexity of the original MINLP problem, at the expense of obtaining sub-optimal solutions. This algorithm computes a sequence of lines to be removed from service, one at the time, until no further decrease in the dispatch cost can be obtained. It identifies the line candidate for removal at each step by exploiting the (continuously relaxed value of) lines breaker statuses at the solution of a relaxed OPF problem. The algorithm relies on solving a sequence of OPF problems formulated as nonlinear programs (NLPs).

The rest of this paper is organized as follows. Section II presents the AC OPF MINLP formulation of the OTS problem. Section III describes the proposed heuristic algorithm for finding a sequence of lines to be removed from service. Section IV provides numerical results with the proposed heuristic algorithm and with a full-fledged MINLP approach on the widely used benchmark reference for this research topic, the IEEE118-bus system. Section V concludes and provides directions for future work.

\section{OTS PROBLEM FORMULATION AS A MINLP}

Let $N$ denote the set of nodes, $G$ the set of generators, and $L$ the set of lines.

The optimization problem is formulated relying on rectangular coordinates of complex voltages:

$$
\underline{V}_{i}=e_{i}+j f_{i}, \quad V_{i}=\sqrt{e_{i}^{2}+f_{i}^{2}}, \quad i \in N,
$$


where $e_{i}$ and $f_{i}$ are the real and imaginary parts respectively, and $V_{i}$ is the voltage magnitude.

The goal of the optimization problem is to minimize the generation dispatch $\operatorname{cost}^{1}$ :

$$
h=\min _{P_{g i}, Q_{g i}, s_{i j}, e_{i}, f_{i}} \sum_{i \in G} c_{g i} P_{g i}
$$

where $P_{g i}$ denotes the active power of generator $i$, and $c_{g i}$ is its cost.

The OTS problem is subject to the following constraints:

$$
\begin{aligned}
& P_{g i}-P_{c i}=\sum_{j \in N} s_{i j} P_{i j}=\sum_{j \in N} s_{i j} g_{i j} V_{i}^{2} \\
& -\sum_{j \in N} s_{i j}\left[\left(e_{i} e_{j}+f_{i} f_{j}\right) g_{i j}+\left(f_{i} e_{j}-e_{i} f_{j}\right) b_{i j}\right], \quad i \in N \\
& Q_{g i}-Q_{c i}+b_{i}^{s h} V_{i}^{2}=\sum_{j \in N} s_{i j} Q_{i j}=-\sum_{j \in N} s_{i j}\left(b_{i j}^{s h}+b_{i j}\right) V_{i}^{2} \\
& +\sum_{j \in N} s_{i j}\left[\left(e_{i} e_{j}+f_{i} f_{j}\right) b_{i j}-\left(f_{i} e_{j}-e_{i} f_{j}\right) g_{i j}\right], \quad i \in N \\
& P_{g i}^{\min } \leq P_{g i} \leq P_{g i}^{\max }, \quad i \in G \\
& Q_{g i}^{\min } \leq Q_{g i} \leq Q_{g i}^{\max }, \quad i \in G \\
& V_{i \min }^{2} \leq e_{i}^{2}+f_{i}^{2} \leq V_{i \max }^{2}, \quad i \in N, \\
& \left(g_{i j}^{2}+b_{i j}^{2}\right)\left[V_{i}^{2}+V_{j}^{2}-2\left(e_{i} e_{j}+f_{i} f_{j}\right)\right] \leq s_{i j}\left(I_{i j}^{\max }\right)^{2} \\
& +K_{I}\left(1-s_{i j}\right), i j \in L \\
& s_{i j} \in\{0,1\}, \quad i j \in L,
\end{aligned}
$$

where, for the generator at bus $i, P_{g i}$ is its active power lying within physical limits $P_{g i}^{\min }$ and $P_{g i}^{\max }, Q_{g i}$ is its reactive power lying within physical limits $Q_{g i}^{\min }$ and $Q_{g i}^{\max }, P_{c i}$ and $Q_{c i}$ are the active and reactive powers of the load at node $i$, $s_{i j}$ is a binary variable that models the on/off status of the line linking nodes $i$ and $j, P_{i j}$ and $Q_{i j}$ are the active and reactive power flows between nodes $i$ and $j, g_{i j}$ and $b_{i j}$ are the conductance and susceptance of the branch linking nodes $i$ and $j, b_{i j}^{s h}$ is the shunt susceptance of the branch linking nodes $i$ and $j, b_{i}^{s h}$ is the shunt bank susceptance at bus $i, V_{i \min }^{2}$ and $V_{i \max }^{2}$ are operational limits of voltage at bus $i,\left(I_{i j}^{\max }\right)^{2}$ is the operational limit of current in the branch linking nodes $i$ and $j$, and $K_{I}$ is a "bigM"-type constant properly chosen to relax constraints (7), if line $i j$ is open at the optimal solution, a fact which is obtained as a result of the optimization.

Constraints (2) and (3) are the AC power flow equations. Constraints (4) and (5) are limits on the generators' active and reactive power output. Constraints (6) are bounds on voltage magnitudes. Constraints (7) are limits on the branch currents. Constraints (8) express the fact that line statuses can take only binary values.

To simplify the mathematical model phase shifter and onload tap changing transformers equations have not been shown

\footnotetext{
${ }^{1} \mathrm{~A}$ linear cost function has been chosen to facilitate benchmarking and comparisons, as such costs are freely available [12] and used in different research works. However, the optimization problem can accommodate straightforwardly other typical cost functions such as nonlinear generation cost curves.
}

explicitly, but they can be written likewise. It is also possible to extend the formulation to take into account multiple parallel lines, and/or to restrict the optimisation problem to a particular subset of "switchable" lines. Furthermore, in principle, one could add as well a constraint limiting the number of disconnected lines at the optimal solution.

\section{Proposed Heuristic Algorithm}

The proposed algorithm uses a greedy approach in order to compute a sequence of lines to be removed from operation, one at a time, until no further (significant) decrease in the dispatch cost can be obtained. At each step, it identifies the line candidate for removal by exploiting the (continuously relaxed value of) line statuses at the solution of a relaxed OPF problem. The algorithm relies on the heuristic observation that the smaller the value of the relaxed status of a line, the larger the expected cost reduction when taking this line out of operation; in particular no cost reduction is to be expected if the relaxed line status is equal to one at the optimum of the relaxed OPF problem.

The main steps of the algorithm are described hereafter:

0) Initialization.

Set the subset of disconnected lines $L_{d}=\emptyset$.

1) Solve the original OPF problem (1)-(8) with all lines in service (i.e. $s_{i j}=1, \quad i j \in L$ ).

Let $h_{c}$ be the current optimal dispatch cost (1).

2) Solve a relaxation of the original OPF problem (1)-(8) augmented with one additional constraint.

This relaxed OPF problem consists of optimizing (1) subject to the following constraints: (2)-(7), continuous relaxation constraints $(9)$ which replace the binary variables constraints (8):

$$
0 \leq s_{i j} \leq 1, i j \in L \backslash L_{d},
$$

equality constraints (10) for the lines already set out of operation at the previous steps:

$$
s_{i j}=0, i j \in L_{d},
$$

and one constraint (11) that enforces the (soft) removal of one additional line:

$$
\sum_{i j \in L \backslash L_{d}} s_{i j}=\left|L \backslash L_{d}\right|-1 .
$$

Let $h_{c}^{\star}$ be the objective of this relaxed OPF problem.

3) Check the first termination criterion.

If the objective of the relaxed OPF problem $h_{c}^{\star}$ is not significantly smaller than the current dispatch cost $h_{c}$ (i.e. if $h_{c}^{\star}>h_{c}-\varepsilon$, where $\varepsilon>0$ is a user chosen termination tolerance) then the algorithm terminates.

4) Determine the next line candidate for removal.

a) If, at the solution of the relaxed OPF problem, all lines status $s_{i j}$ 's are integer, select (the single) line $d$ which has the status equal to zero. Go to step $4 \mathrm{~d}$.

b) Rank the lines for which $s_{i j}<1$ in increasing order of their status. 
c) Disconnect the top ranked line and, for this fixed network topology, solve the OPF problem (1)-(7). Repeat ${ }^{2}$ this step for all lines from the ranking.

d) Let $d$ be the best tested line to disconnect (i.e. the line that leads to the smallest dispatch cost among all tested lines) and let $h_{d}$ be its corresponding dispatch cost.

e) Check for false alarms: if the best line has a low value of $s_{i j}$ (e.g. $s_{i j} \leq 0.05$ ) and the objective of the relaxed OPF is significantly better than the current cost dispatch (e.g. $h_{c}^{\star}<0.9 h_{c}$ ) but at the same time the corresponding $h_{d}$ does not improve the current dispatch cost (i.e. $h_{d} \geq h_{c}$ ), then force the corresponding $s_{i j}=1$ for all subsequent iterations.

Go back to step 2.

5) Check the second termination criterion.

If the dispatch cost $h_{d}$ obtained by disconnecting the best tested line is not significantly smaller than the current dispatch cost $h_{c}$ (i.e. if $h_{c}-h_{d} \leq \varepsilon$, where $\varepsilon>0$ is a user chosen termination tolerance) then the algorithm terminates.

6) Updating step.

Update the set of connected lines $L=L \backslash\{d\}$.

Update the set of disconnected lines $L_{d}=L_{d} \cup\{d\}$ and fix disconnected lines status $s_{i j}=0, i j \in L_{d}$. Update the current dispatch cost $h_{c} \leftarrow h_{d}$.

Go to step 2.

Note that all OPF problems involved in this algorithm in steps 1,2 , and $4 \mathrm{c}$ have only continuous variables. The computational effort of the algorithm is proportional with the number of OPF runs in steps 2 and 4c.

\section{ILlustrative EXAMPLES}

\section{A. Considerations}

To provide benchmark results and facilitate comparison, the proposed approach is applied on the IEEE118 system, which has been widely used for the OTS problem [9], [10], [11], [12], [13], [14]. The main data of the system have been taken from [15], except for generator cost functions and thermal branch limits, which have been taken from [12]. It was further assumed that 35 generators that do not produce initially active power are treated as synchronous compensators. The system contains 118 buses, 118 loads, 177 lines, 19 generators, 35 synchronous compensators, 9 transformers, 14 shunt banks. Voltage limits are set to 0.94 p.u. and 1.06 p.u. at all buses.

Three load levels are considered hereafter:

- normal load which corresponds to the original system load level (i.e. 4242 MW and 1438 MVAr);

- high load scenario where each load is increased with $10 \%$ above the normal load demand;

\footnotetext{
${ }^{2}$ To speed up computations lines for which relaxed values of the breaker status are too high are skipped (e.g by discarding all lines such that $s_{i j}>0.5$, at most two lines will be tested at each iteration).
}

TABLE I

OPTIMAL COST OF GENERATION DISPATCH WITH FIXED NETWORK TOPOLOGY

\begin{tabular}{|c|c|}
\hline load level & overall cost $(\$ / h)$ \\
\hline normal & 1683.38 \\
\hline high & 2672.79 \\
\hline very high & 4805.91 \\
\hline
\end{tabular}

TABLE II

MINLP APPROACH: COST SAVINGS AND LINES REMOVED FOR THE THREE LOAD SCENARIOS

\begin{tabular}{|c|c|c|}
\hline $\begin{array}{l}\text { load } \\
\text { level }\end{array}$ & $\begin{array}{c}\text { cost reduction } \\
(\%)\end{array}$ & $\begin{array}{c}\text { lines removed } \\
\text { (bus to bus) }\end{array}$ \\
\hline normal & 4.82 & $\begin{array}{c}11-13,23-34,45-46,47-49,48-49,54-55 \\
54-56,55-56,56-58,60-62,61-62,24-72, \\
74-75,76-77,78-79,85-89,109-110,32-114\end{array}$ \\
\hline high & 10.51 & $\begin{array}{c}46-47,47-49,52-53,54-55,55-56,56-57 \\
56-58,62-66,62-67,49-69,70-75,74-75 \\
78-79,32-114\end{array}$ \\
\hline $\begin{array}{l}\text { very } \\
\text { high }\end{array}$ & 17.60 & $\begin{array}{c}12-16,19-20,46-47,47-49,52-53,49-54, \\
49-54,55-56,51-58,60-62,61-62,49-69, \\
70-75,74-75,78-79,100-103,32-113,32-114\end{array}$ \\
\hline
\end{tabular}

- very high load scenario where each load is increased with $20 \%$ above the normal load demand.

The MINLP and NLP optimization problems have been developed in GAMS version 23.9.3 [16] and solved using the BONMIN solver and the IPOPT solver, respectively. All tests have been performed on a PC of 2.8-GHz and 4-Gb RAM.

\section{B. OPF solutions with fixed topology}

To foster benchmark comparison, Table I provides the optimal cost of generation dispatch obtained for fixed network topology in the three load scenarios. One can observe that the cost increases sharply with the load level. For each load level the thermal limits of the same lines (i.e. 30-38, 65-68, and 69-77) are binding at the optimum.

\section{MINLP solutions}

Despite the huge combinatorial space of this problem (i.e., $2^{177}$ possible states stemming from the on/off status of 177 lines), unexpectedly, the original MINLP problem (1)-(8) has been solved in a relatively short computational time (10 minutes in the slowest case) providing very good quality solutions, as a very tight value of $0.01 \%$ has been used for the relative optimality gap.

Table II provides the cost savings and the lines removed at the solution provided by the MINLP solver. One can observe that the larger the load level, the higher the cost savings, and that the cost savings are significant in all cases. However, an important number of lines are removed in all cases (e.g. 18 lines for normal and very high load levels, and 14 lines for the high load level).

For each load level the same lines (i.e. 65-68 and 69-77) present binding thermal limits at the optimum.

\section{Proposed heuristic approach versus MINLP approach}

Table III provides the cost savings, the number of lines removed, and the CPU times for both the proposed heuristic 
TABLE III

PROPOSED HEURISTIC APPROACH VERSUS MINLP APPROACH: COST SAVINGS AND LINES REMOVED FOR THE THREE LOAD SCENARIOS

\begin{tabular}{|c|c|c|c|c|c|c|}
\hline $\begin{array}{c}\text { load } \\
\text { level }\end{array}$ & \multicolumn{2}{|c|}{ cost reduction (\%) } & \multicolumn{2}{c|}{$\begin{array}{c}\text { number of } \\
\text { lines removed }\end{array}$} & \multicolumn{2}{c|}{ CPU time (s) } \\
& MINLP & heuristic & MINLP & heuristic & MINLP & heuristic \\
\hline normal & 4.82 & 4.03 & 18 & 3 & 637 & 17 \\
\hline high & 10.51 & 9.14 & 14 & 4 & 264 & 26 \\
\hline very high & 17.60 & 4.61 & 18 & 5 & 428 & 27 \\
\hline
\end{tabular}

approach and the MINLP approach. In the proposed approach we have used the following values of the parameters: i) for the selection of candidate lines (step 4.c), we use a threshold of 0.5 on $s_{i j}$ gotten from the relaxed OPF solution; ii) we use the threshold value $\varepsilon=1 \$ / h$ as concerns the termination criteria on $h_{c}-h_{d}$ and $h_{c}-h_{c}^{\star}$; iii) we use a line status threshold $s_{i j}=0.05$ for detecting the false alarms (step $4 \mathrm{e}$ ).

Compared to the MINLP approach, the cost reduction achieved with the proposed heuristic method is very good for the normal and high load scenarios (i.e. $16.4 \%$ and $13.03 \%$ less than the MINLP), and average (i.e. $73.8 \%$ less than the MINLP) for the very high load scenario. However, the cost reduction in the proposed approach is obtained by using a significantly smaller number of lines, which is easier to implement and accept by a transmission system operator. In particular for the normal load level the removal of the 3 lines proposed by the heuristic approach (see Table IV) has only a slightly less efficient effect than the opening of the 18 lines proposed by the MINLP approach. Furthermore, the proposed heuristic approach has the advantage of providing the sequence of lines to be removed whereas the MINLP provides only the final optimal topology but gives no clue in which order the lines should be removed from service. Finally, and very importantly, the proposed approach is much faster and scales much better than the MINLP as it requires successive solutions of OPF problems with continuous variables only, while MINLP is well-known to scale badly with the problem size and number of binary variables, being therefore an utopian approach for larger real-world systems.

\section{E. Detailed results with the proposed heuristic approach}

Table IV and Figures 1, 2, 3 provide, for the three load levels considered, the sequence of lines opened by the proposed approach as well as the cost saving as a function of the number of lines removed. From Figures 1 and 3 one can observe that for the normal and very high load levels the impact of opening an additional line on cost savings is almost constant. On the other hand, for the high load level (see Fig. 2), the removal of the first two lines lead to a much smaller cost reduction compared to the third and especially the fourth lines removed.

1) Results for the normal load level: Table $V$ yields, at each iteration of the proposed algorithm (iteration 0 corresponds to the case where all lines are in service), the status of the best candidates lines for disconnection at the solution of the OPF relaxation and the OPF cost obtained with the line taken out from service. The lines proposed for opening by
TABLE IV

SEQUENCE OF LINES REMOVED BY THE PROPOSED HEURISTIC ALGORITHM FOR THE THREE LOAD SCENARIOS

\begin{tabular}{|c|c|c|c|}
\hline nb & \multicolumn{3}{|c|}{ load level } \\
iter & normal & high & very high \\
\hline 1 & $76-77$ & $62-66$ & $62-66$ \\
2 & $24-70$ & $66-67$ & $66-67$ \\
3 & $24-72$ & $24-72$ & $49-54$ \\
4 & & $24-70$ & $50-57$ \\
5 & & & $49-51$ \\
\hline
\end{tabular}

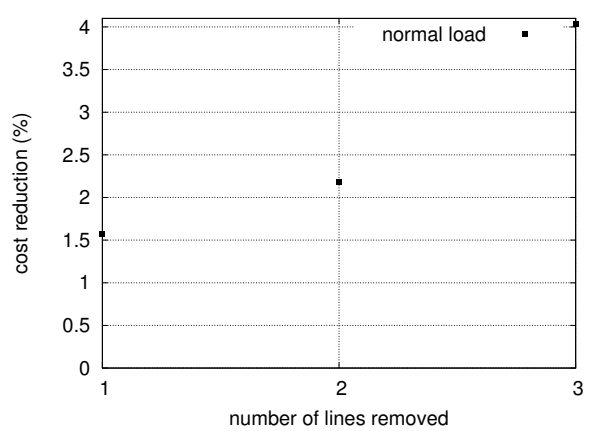

Fig. 1. Normal load scenario: cost reduction versus number of lines opened

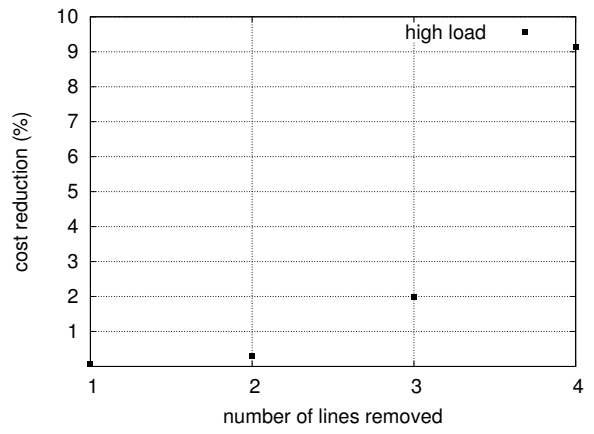

Fig. 2. High load scenario: cost reduction versus number of lines opened

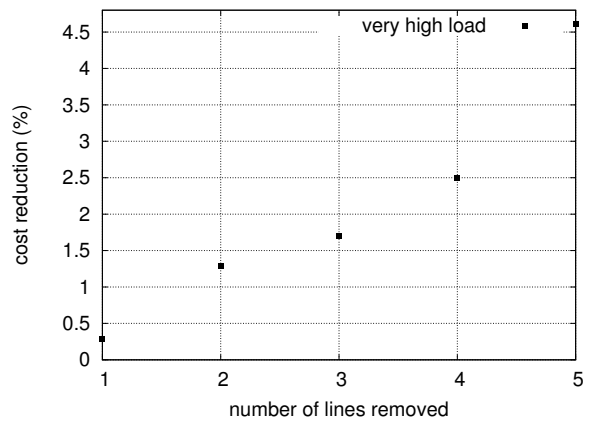

Fig. 3. Very high load scenario: cost reduction versus number of lines opened 
TABLE V

RESULTS WITH THE PROPOSED HEURISTIC ALGORITHM FOR THE NORMAL LOAD SCENARIO

\begin{tabular}{|c|c|c|c|}
\hline iter & line & status & cost $(\$ / h)$ \\
\hline 0 & - & - & 1683.38 \\
1 & $\mathbf{7 6 - 7 7}$ & $\mathbf{0 . 0 0 0}$ & $\mathbf{1 6 5 6 . 9 7}$ \\
2 & $\mathbf{2 4 - 7 0}$ & $\mathbf{0 . 0 0 0}$ & $\mathbf{1 6 4 6 . 6 8}$ \\
3 & $\mathbf{2 4 - 7 2}$ & $\mathbf{0 . 0 0 0}$ & $\mathbf{1 6 1 5 . 5 3}$ \\
4 & $68-81$ & 0.454 & 1636.89 \\
& $49-69$ & 0.546 & 1621.72 \\
\hline
\end{tabular}

the algorithm, and their corresponding results, are shown with bold characters.

Note that, in the first three iterations the OPF relaxation provides an integer solution, as the status of each line is equal to 0 . Furthermore, opening each of the proposed lines reduces the dispatch cost. At the fourth iteration there are two lines (68-81 and 49-69) for which the status value is neither 0 or 1. As opening any of these two lines leads to an increase of the dispatch cost the heuristic algorithm stops, the proposed dispatch cost being the one obtained at the third iteration. The algorithm requires overall solving six relaxed OPF problems.

The results obtained at the fourth iteration suggest that if the relaxed lines status are not sufficiently close to zero these lines may not be effective candidate to reduce the dispatch cost.

2) Results for the high load level: Table VI provides the main results at the solution of the OPF relaxation at each iteration of the proposed algorithm.

Note that in the first iteration although line 14-15 has a very small value of its relaxed status it does not lead to a cost reduction. This line is a tricky false alarm due to the fact that the relaxed OPF problem provides a very low but purely utopian lower bound $\left(h_{c}^{\star}=1771.78 \$ / h\right)$, masking thereby other potential good lines candidates. This false alarm is identified at the step $4 \mathrm{e}$ of the proposed algorithm and its status is fixed to 1 for subsequent steps. Then, for the same reason, the algorithm identifies line 12-16 as a false alarm. Next, the algorithm finds the line 62-66 as best candidate, which leads indeed at a cost reduction. The algorithm proceeds and stops at iteration 5 because, for line 15-33, the cost increases and the relaxed value of its status (0.065) is higher than the false alarm status threshold which was set to 0.05 .

Compared with the solutions obtained for the normal load level (see Table V), in this case the OPF relaxation never provides an integer solution.

The algorithm requires overall solving eleven relaxed OPF problems.

3) Results for the very high load level: Table VII provides the main results at the solution of the OPF relaxation at each iteration of the proposed algorithm.

Note that in the first iteration four false alarms lines (see step $4 \mathrm{e}$ of the proposed algorithm), with very small values of the status and significantly smaller value of the relaxed OPF objective with respect to the current dispatch cost, are checked successively before obtaining a cost reduction. Also, in two cases the proposed best lines for opening lead to infeasible
TABLE VI

RESULTS WITH THE PROPOSED HEURISTIC ALGORITHM FOR THE HIGH LOAD SCENARIO

\begin{tabular}{|c|c|c|c|}
\hline iter & line & status & cost $(\$ / h)$ \\
\hline 0 & - & - & 2672.79 \\
1 & $14-15$ & 0.027 & 2673.71 \\
& $12-16$ & 0.027 & 2675.47 \\
& $\mathbf{6 2 - 6 6}$ & $\mathbf{0 . 0 2 6}$ & $\mathbf{2 6 7 0 . 8 1}$ \\
2 & $12-14$ & 0.027 & 2674.33 \\
& $\mathbf{6 6 - 6 7}$ & $\mathbf{0 . 0 2 6}$ & $\mathbf{2 6 6 5 . 1 1}$ \\
3 & $\mathbf{2 4 - 7 2}$ & $\mathbf{0 . 0 2 7}$ & $\mathbf{2 6 1 9 . 9 3}$ \\
4 & $\mathbf{2 4 - 7 0}$ & $\mathbf{0 . 0 2 9}$ & $\mathbf{2 4 2 8 . 2 8}$ \\
5 & $49-69$ & 0.029 & 2482.61 \\
& $15-19$ & 0.029 & 2482.74 \\
& $16-17$ & 0.029 & 2485.75 \\
& $15-33$ & 0.065 & 2484.95 \\
\hline
\end{tabular}

TABLE VII

RESULTS WITH THE PROPOSED HEURISTIC ALGORITHM FOR THE VERY HIGH LOAD SCENARIO

\begin{tabular}{|c|c|c|c|}
\hline iter & line & status & cost $(\$ / h)$ \\
\hline 0 & - & - & 4805.91 \\
1 & $14-15$ & 0.017 & 4813.13 \\
& $12-16$ & 0.017 & 4821.58 \\
& $11-13$ & 0.127 & infeasible \\
& $12-14$ & 0.017 & 4885.86 \\
& $\mathbf{6 2 - 6 6}$ & $\mathbf{0 . 0 1 7}$ & $\mathbf{4 7 9 1 . 8 5}$ \\
2 & $\mathbf{6 6 - 6 7}$ & $\mathbf{0 . 0 1 7}$ & $\mathbf{4 7 4 3 . 8 9}$ \\
3 & $\mathbf{4 9 - 5 4}$ & $\mathbf{0 . 0 1 7}$ & $\mathbf{4 7 2 4 . 3 2}$ \\
4 & $\mathbf{5 0 - 5 7}$ & $\mathbf{0 . 0 1 7}$ & $\mathbf{4 6 8 6 . 3 8}$ \\
5 & $\mathbf{4 9 - 5 1}$ & $\mathbf{0 . 0 1 7}$ & $\mathbf{4 5 8 4 . 2 5}$ \\
6 & $15-19$ & 0.493 & infeasible \\
& $32-114$ & 0.525 & 4584.43 \\
\hline
\end{tabular}

OPF problems.

The algorithm requires in this case solving eleven relaxed OPF problems.

\section{CONCLUSIONS AND FUTURE WORKS}

Unlike most existing approaches, which investigate the problem of optimal transmission switching for generators dispatch cost reduction relying on a questionnable linear DC grid model, this paper has proposed, relying on the accurate AC OPF model, a new heuristic algorithm aimed to provide a sequence of lines to be disconnected in order to decrease generators dispatch cost.

The proposed algorithm requires the solution of various NLP OPF problems and therefore is computationally efficient and scalable to systems of large size. Results on the IEEE118bus system show that the approach provides good quality suboptimal solutions with relatively small computational effort by removing only a few lines from service. The latter feature of the algorithm is of practical importance because, due to practical needs (e.g. cost of breaker maintenance, decreasing cost reduction effectiveness as the number of switching operations grows, coupling topologies between successive operating points, system topology does not deviate too much from the known usual topology, etc.) system operators generally look for a few effective lines switching.

In order to leverage the approach to a potential use by system operators the present work as well as the previous works on this subject should be further extended to provide 
solutions which satisfy: the $\mathrm{AC} \mathrm{N}-1$ contingency reliability criterion (a prerequisite requirement to take into account is that the optimized topology does not lead to islands for any N-1 postulated contingency), fault constraints (including possible adjustment of protection settings), and stability constraints.

However, most importantly, further research is needed to ascertain whether the reduction of dispatch cost through optimal transmission switching is also valid outside the widely used IEEE118-bus test system (or the likewise IEEE standard test systems) [15].

\section{ACKNOWLEDGEMENTS}

This paper presents research results of the Belgian Network DYSCO, funded by the Interuniversity Attraction Poles Programme, initiated by the Belgian State. The scientific responsibility rests with the authors.

\section{REFERENCES}

[1] J.G. Rolim and L.J.B. Machado, "A study of the use of corrective switching in transmission systems", IEEE Trans. Power Syst., Vol. 14, No. 1, 1999, pp. 336-341.

[2] F. Capitanescu, J.L. Martinez Ramos, P. Panciatici, D. Kirschen, A Marano Marcolini, L. Platbrood, and L. Wehenkel, "State-of-the-art, challenges, and future trends in security-constrained optimal power flow", Elect. Power Syst. Research, vol. 81, no. 8, pp 1731-1741, 2011.

[3] K.W. Hedman, S.S. Oren, and R.P. O'Neill, "A Review of Transmission Switching and Network Topology Optimization", IEEE PES General Meeting, Detroit (USA), 2011.

[4] E. Lobato, F. Echavarren, L. Rouco, M.I. Navarrete, R. Casanova, G. Lopez, "A Mixed-Integer LP Based Network Topology Optimization Algorithm for Overload Alleviation", PowerTech Conference, Bologna (Italy), 2003.
[5] W. Shao and V. Vittal, "Corrective switching algorithm for relieving overloads and voltage violations", IEEE Trans. Power Syst., Vol. 20, No. 4, 2005, pp. 1877-1885.

[6] R. Bacher and H. Glavitsch, "Loss reduction by network switching", IEEE Trans. Power Syst., Vol. 3, No. 2, 1988, pp. 447-454.

[7] S. Fliscounakis, F. Zaoui, G. Simeant, and R. Gonzalez, "Topology Influence on Loss Reduction as a Mixed Integer Linear Programming Problem", Proc. of IEEE Powertech Conference, Lausanne, Switzerland, 2007.

[8] F. Zaoui, S. Fliscounakis, and R. Gonzalez, "Coupling OPF and Topology Optimization for Security Purposes", Proc. of PSCC Conference, Liège, Belgium, 2005.

[9] E.B. Fisher, R.P. O'Neill, M.C. Ferris, "Optimal transmission switching", IEEE Trans. Power Syst., vol. 23, no. 3, 2008, pp. 1346-1355.

[10] K.W. Hedman, R.P. O'Neill, E.B. Fisher, S.S. Oren, "Optimal transmission switching with contingency analysis", IEEE Trans. Power Syst., Vol. 24, No. 3, 2009, pp. 1577-1586.

[11] J.D. Fuller, R. Ramasra, and A. Cha, "Fast Heuristics for TransmissionLine Switching”, IEEE Trans. Power Syst., vol. 27, no. 3, 2012, pp. $1377-$ 1386.

[12] S. Blumsack, "Network topologies and transmission investment under electric-industry restructuring", Ph.D. dissertation, Dept. Eng. and Public Policy, Carnegie Mellon University, Pittsburgh, PA, 2006.

[13] T. Potluri and K. Hedman, "Impacts of topology control on the ACOPF", IEEE PES General Meeting, San Diego (USA), 2012.

[14] M. Soroush and J.D. Fuller, "Accuracies of Optimal Transmission Switching Heuristics Based on DCOPF and ACOPF", IEEE Trans. Power Syst., vol. 29, no. 2, 2014, pp. 924-932.

[15] R. D. Zimmerman, C. E. Murillo-Sanchez, and R. J. Thomas, "MATPOWER: Steady-State Operations, Planning and Analysis Tools for Power Systems Research and Education", IEEE Trans. Power Syst., vol. 26, no. 1, pp. 12-19, Feb. 2011.

[16] B.A. McCarl, "GAMS User Guide", Version 23.8, 2012. Available online: www.gams.com. 